\title{
A REMARK ON TRANSFORMATION GROUPS LEAVING FIXED AN END POINT
}

\author{
HSIEN-CHUNG WANG
}

1. In a recent paper [2], Wallace asked, among other questions, the following one: Let $G$ be a compact transformation group ${ }^{1}$ of a Peano continuum $S$ leaving fixed an end point of $S$. Must G have some other fixed points? If $G$ is assumed to be cyclic instead of compact, Wallace [1] proved that $G$ must have another fixed point. It is the aim of the present remark to answer the above question in the affirmative under weaker condition. In fact, we prove the following results.

THEOREM. Let $G$ be a transformation group of an arcwise connected, Hausdorff space $X$ leaving fixed an end point $e$ of $X$. Then $G$ has no other fixed point if and only if, given any neighborhood $U$ of $e$, the orbit $G(U)$ under $G$ coincides with the whole space $X$.

Corollary. Let $G, X$, e have the same meaning as in the theorem. If $G$ is, moreover, compact, then $G$ has a fixed point other than $e$.

2. Let $X$ be a connected, Hausdorff space. A point $e$ of $X$ is called an end point of $X$ if, given any neighborhood $U$ of $e$, there exists a point $x$ of $U$ such that $X-x=X_{1} \cup X_{2}$ separate and that $e \in X_{1} \subset U$ $[3$, p. 82]. From now on, we shall always assume $X$ to be arcwise connected and $e$ an end point of $X$. Let $Y$ be a closed subset of $X$ not containing $e$. We shall construct a point $q$ which is uniquely determined, in a topological manner, by the pair $(e, Y)$.

Let $C$ be the set of all points which separate $e$ and $Y$. Since $Y$ is closed and $e$ an end point, the set $C$ is not empty and has $e$ as one of its limiting points. Let $y$ be a point of $Y$, and $l=l(t)$ be an arc joining $e$ and $y$ with $l(0)=e, l(1)=y$. Evidently, $C$ is a subset of $l$. The natural ordering of points of $l$ by the parameter $t$ gives an ordering of points of $C$, and this ordering is linear. More precisely, for any two points $c_{1}=l\left(t_{1}\right), c_{2}=l\left(t_{2}\right)$ of $C$, we say $c_{1}<c_{2}$ whenever $t_{1}<t_{2}$. Denote by $q=l\left(t_{0}\right)$ the upper limit of $C$ in $l$. Evidently, the point $q$ exists and belongs to $l$. From the construction it seems that the point $q$ depends on $y$ as well as the arc $l$. However, this is not so, as shown by the following lemma.

Presented to the Society, September 7, 1951, under the title $A$ theorem on fixed points under a group of homeomorphisms; received by the editors December 10, 1951.

${ }^{1}$ Here, by a transformation group $G$ of $X$, we mean (i) $G$ is an abstract group of homeomorphisms of $X$ onto itself, and (ii) $G$ is a topological space such that the function $f: G \times X \rightarrow X$ defined by $f(g, x)=g(x), g \in G, x \in X$, is continuous. 
Lemma. The point $q$ is the only point in $\bar{C}$ such that any $c \in C$, with $c \neq q$, separates $e$ and $q$. Thus $q$ is a topological invariant of the pair $(e, Y)$.

Proof. Let $c=l\left(t^{\prime}\right)$ be a point of $C$ and $c \neq q$. Then there exist disjoint open sets $V_{1}, V_{2}$ such that

$$
X-c=V_{1} \cup V_{2}, \quad e \in V_{1}, \quad Y \subset V_{2} .
$$

By definition of $q$, we know that $t^{\prime}<t_{0}$. Thus the arc $l(t), t_{0} \leqq t \leqq 1$, joins $q$ and $y$ in $X-c$. Hence $q \in V_{2}$, and hence $c$ separates $e$ and $q$.

Conversely, let $q^{*}=l\left(t^{*}\right)$ be a point of $\bar{C}$ with the property that each point $c$ of $C$ with $c \neq q^{*}$ separates $e$ and $q^{*}$. Evidently, $t^{*} \leqq t_{0}$. Suppose that $t^{*}<t_{0}$. There exists then a point $c^{\prime}=l(s)$ of $C$ with $t^{*}<s$. The arc $l(t), 0 \leqq t \leqq t^{*}$, joins $e$ and $q^{*}$ in $X-c^{\prime}$. Thus $c^{\prime}$ does not separate $e$ and $q^{*}$. A contradiction is obtained. In other words, $t^{*}=t_{0}$ and $q^{*}=q_{0}$. The lemma is proved.

3. Now we can prove our theorem quite easily. Let $G$ be a transformation group of $X$ leaving fixed an end point $e$. Suppose that, for each neighborhood $U$ of $e$, we have $G(U)=X$. It follows from the separation axiom that $G$ cannot have any fixed point different from $e$. Conversely, suppose that there exists a neighborhood $U$ of $e$ such that $G(U) \neq X$. Since $U$ is open, the orbit $G(U)$ is also open. Hence $Y=X-G(U)$ is closed. The lemma tells us that there exists a point $q, q \neq e$, which is uniquely determined, in a topological manner, by the pair $(e, Y)$. For each transformation $g$ of $G$, we have $g(e)=e$, $g(Y)=Y$. It follows that $g(q)=q$. Hence $q$ is a fixed point of $G$. The theorem is hereby proved.

Using known results $[3, \S 11]$ it is possible to shorten slightly the argument given above and, indeed, to define $q$ in a topological manner $\mathrm{ab}$ initio. But the above proof is deemed preferable since it is quite self-contained and brief.

Proof of the Corollary. Take a point $x$ different from $e$. Since $G$ is compact, the orbit $G(x)$ is closed in $X$. The set $U=X-G(x)$ is, therefore, a neighborhood of $e$ with $G(U)=U \neq X$. By the above theorem, $G$ has a fixed point other than $e$.

\section{REFERENCES}

1. A. D. Wallace, A fixed-point theorem, Bull. Amer. Math. Soc. vol. 51 (1945) pp. $413-416$.

2. - Group invariant continua, Fund. Math. vol. 36 (1949) pp. 119-124.

3. R. L. Wilder, Topology of manifolds, Amer. Math. Soc. Colloquium Publications, vol. 32, New York, 1949.

Institute for Advanced Study 